\title{
MIXED LINEAR INTEGRAL EQUATIONS OF THE FIRST ORDER*
}

\author{
BY
}

\section{WALLIE ABRAHAM HURWITZ}

In a previous paper, $\dagger$ the author has defined a mixed $\ddagger$ integral equation as an equation in which an unknown function may be involved under integral signs operating over regions of different numbers of dimensions and in which the value of the function at special points may occur. The highest number of dimensions of any integral was called the order of the equation. A theorem was proved concerning mixed linear equations of the first order. It is the purpose of the present paper to give a systematic treatment of this case. $\S$ A complete theory of a special form of such equations, together with interesting applications to physical problems, has been given by Kneser.\|

Mixed linear integral equations are of interest, not only because they present themselves naturally in the attempt to solve certain problems in connection with differential equations, but also because they furnish another chapter in the theory of equations involving linear operations. As might be expected, they follow closely the analogy of linear algebraic equations and pure linear integral equations; but the generalizations necessary to exhibit the analogy are sometimes by no means trivial. Thus, a certain lack of symmetry pointed out at the close of the earlier paper has now been removed by the device of considering as adjoint to the given equation (which involves one function as the only unknown) a system of equations, involving one unknown function and several unknown constants.

1. We consider the homogeneous mixed linear integral equation

$$
u(x)=\sum_{i=1}^{m} K^{(i)}(x) u\left(\xi_{i}\right)+\int_{a}^{b} K(x, s) u(s) d s
$$

* Presented to the Society, December 31, 1912.

† Bulletin of the American Mathematical Society, vol. 18 (1912), p. 291.

$\ddagger$ The term "mixed equation" has been used in a different sense by Bratu, P a r i s C o m pt e s $R$ e n d u s, vol. 148 (1909), p. 1370.

$\S$ The next higher case, in which appear integrals over two- and one-dimensional regions, as well as values of the function at special points, has been studied by Dr. J. Rosenbaum, in a paper to be published soon.

II Rendicontidel Circolo Matematico di Palermo, vol. 37 (1914), p. 169. In terms of the notation of the present paper, the special case considered by Kneser is that in which $K^{(i)}(x)=M_{i} K\left(x, \xi_{i}\right)$, where the quantities $M_{i}$ are positive constants. 
and the non-homogeneous equation

$$
u(x)=f(x)+\sum_{i=1}^{m} K^{(i)}(x) u\left(\xi_{i}\right)+\int_{a}^{b} K(x, s) u(s) d s .
$$

The points $\xi_{1}, \xi_{2}, \cdots, \xi_{m}$ are any distinct points of the interval $a \leqq x \leqq b$. The given functions $f(x), K^{(1)}(x), \cdots, K^{(m)}(x)$ will be assumed to be continuous, $a \leqq x \leqq b$; and the function $K(x, y)$ will be assumed to be continuous, $a \leqq x \leqq b, a \leqq y \leqq b$. We speak of the set of functions

$$
\left[K(x, y) ; K^{(1)}(x), \cdots, K^{(m)}(x)\right]
$$

as the kernel system of the equations (1), (2). By a solution $u(x)$ we shall understand without further specification a continuous solution. The solution $u(x)=0$ of (1) will be termed a trivial solution.

It is convenient to consider with equations (1), (2) the adjoint homogeneous system of equations

$$
\begin{aligned}
& v(y)=\sum_{i=1}^{m} v^{(i)} K\left(\xi_{i}, y\right)+\int_{a}^{b} v(s) K(s, y) d s, \\
& v^{(p)}=\sum_{i=1}^{m} v^{(i)} K^{(p)}\left(\xi_{i}\right)+\int_{a}^{o b} v(s) K^{(p)}(s) d s \\
& \quad(p=1,2, \cdots, m)
\end{aligned}
$$

and the non-homogeneous system

$$
\begin{aligned}
v(y) & =g(y)+\sum_{i=1}^{m} v^{(i)} K\left(\xi_{i}, y\right)+\int_{a}^{b} v(s) K(s, y) d s, \\
v^{(p)} & =g^{(p)}+\sum_{i=1}^{m} v^{(i)} K^{(p)}\left(\xi_{i}\right)+\int_{a}^{b} v(s) K^{(p)}(s) d s \\
& (p=1,2, \cdots, m) .
\end{aligned}
$$

Here $g(y)$ is a given continuous function, $a \leqq y \leqq b$, and $g^{(1)}, \cdots, g^{(m)}$ are given constants. By a solution is understood a system consisting of a continuous function $v(y)$ and $m$ constants $v^{(1)}, \cdots, v^{(m)}$, satisfying the equations. We term the solution of $\left(1^{\prime}\right)$

$$
v(y)=0, \quad v^{(1)}=\cdots=v^{(m)}=0
$$

a trivial solution. If a number of solutions are so related that a linear combination of them, not all coefficients being equal to zero, yields a trivial solution, they are said to be linearly dependent;* otherwise linearly independent.

Let the normal orthogonal sets of principal solutions for $K(x, y)$ regarded as kernel of a pure integral equation be $\phi_{1}(x), \cdots, \phi_{n}(x) ; \psi_{1}(y), \cdots$, $\psi_{n}(y)$; then

* The same constant multipliers must be used for the functions contained in the solutions as for the sets of constants. 


$$
\begin{array}{rlr}
\phi_{j}(x) & =\int_{a}^{b} K(x, s) \phi_{j}(s) d s \quad(j=1,2, \cdots, n), \\
\psi_{j}(y) & =\int_{a}^{b} \psi_{j}(s) K(s, y) d s \quad(j=1,2, \cdots, n), \\
\int_{a}^{b} \phi_{j}(s) \phi_{q}(s) d s & =\int_{a}^{b} \psi_{j}(s) \psi_{q}(s) d s=\left\{\begin{array}{l}
0, q \neq j . \\
1, q=j .
\end{array}\right.
\end{array}
$$

The case in which no principal solutions exist is included by making the evident interpretation of the symbols involved when $n=0$.

There exists a continuous function $k(x, y)$ such that $\dagger$

$$
\begin{aligned}
& k(x, y)=K(x, y)+\int_{a}^{b} K(x, s) k(s, y) d s-\sum_{j=1}^{n} \psi_{j}(x) \psi_{j}(y), \\
& k(x, y)=K(x, y)+\int_{a}^{b} k(x, s) K(s, y) d s-\sum_{j=1}^{n} \phi_{j}(x) \phi_{j}(y) .
\end{aligned}
$$

The equation

$$
u(x)=f(x)+\int_{a}^{b} K(x, s) u(s) d s
$$

has no solutions unless

$$
\int_{a}^{b} \psi_{j}(s) f(s) d s=0 \quad(j=1,2, \cdots, n) ;
$$

if this condition is satisfied, solutions exist and may be written in the form

$$
u(x)=f(x)+\int_{a}^{b} k(x, s) f(s) d s+\sum_{j=1}^{n} a_{j} \phi_{j}(x),
$$

where $a_{1}, \cdots, a_{n}$ are constants. Similarly the equation

$$
v(y)=g(y)+\int_{a}^{b} v(s) K(s, y) d s
$$

has no solutions unless

$$
\int_{a}^{b} g(s) \phi_{j}(s) d s=0 \quad(j=1,2, \cdots, n) ;
$$

if this condition is satisfied, solutions exist and may be written in the form

$$
v(y)=g(y)+\int_{a}^{b} v(s) k(s, y) d s+\sum_{j=1}^{n} b_{j} \psi_{j}(y)
$$

where $b_{1}, \cdots, b_{n}$ are constants.

2. Theorem I. Any solution $u(x)$ of (1) is expressible in the form

(6) $u(x)=\sum_{i=1}^{m} A_{i}\left[K^{(i)}(x)+\int_{a}^{b} k(x, s) K^{(i)}(s) d s\right]+\sum_{j=1}^{n} B_{j} \phi_{j}(x)$,

† W. A. Hurwitz, these Trans a c tion s, vol. 13 (1912), p. 408. 
where the $m+n$ constants $A_{1}, \cdots, A_{m} ; B_{1}, \cdots, B_{n}$ satisfy the system of $m+n$ linear algebraic equations:

$$
\sum_{i=1}^{m} A_{i} \int_{a}^{b} \psi_{q}(s) K^{(i)}(s) d s=0 \quad(q=1,2, \cdots, n),
$$

$$
-A_{p}+\sum_{i=1}^{m} A_{i}\left[K^{(i)}\left(\xi_{p}\right)+\int_{a}^{b} k\left(\xi_{p}, s\right) K^{(i)}(s) d s\right]+\sum_{j=1}^{n} B_{j} \phi_{j}\left(\xi_{p}\right)=0
$$

$$
(p=1,2, \cdots, m) \text {. }
$$

Conversely, if $A_{1}, \cdots, A_{m} ; B_{1}, \cdots, B_{n}$ satisfy (7), the function $u(x)$ given by (6) is a solution of (1).

To prove the first part of the theorem, suppose that (1) possesses a solution $u(x)$, and write $u\left(\xi_{i}\right)=A_{i}$; we have then

$$
u(x)=\sum_{i=1}^{m} A_{i} K^{(i)}(x)+\int_{a}^{b} K(x, s) u(s) d s .
$$

Conditions (4) yield the first set of equations (7). If we next write out $u(x)$ by use of (5), we have (6), and the substitution of $\xi_{p}$ for $x$ then gives the last set of (7). Conversely, if $\left[A_{1}, \cdots, A_{m} ; B_{1}, \cdots, B_{n}\right]$ represents a solution of (7), then the first set of (7) furnishes a sufficient condition that $u(x)$ should satisfy (8); the second set shows that $u\left(\xi_{p}\right)=A_{p}$, thus identifying (8) with (1).

CoRollary I. The correspondence between solutions of (1) and solutions of (7) is unique.

This statement will be proved if we demonstrate the following:

Corollary II. To a trivial solution of (1) corresponds a trivial solution of (7); and conversely.

That a trivial solution of (7) leads to a trivial solution of (1) is evident from the form of (6). To prove the other part of the proposition, suppose in (6) $u(x)=0$; then as $u\left(\xi_{p}\right)=A_{p}$,

and

$$
A_{p}=0 \quad(p=1,2, \cdots, m),
$$

$$
0=\sum_{j=1}^{n} B_{j} \phi_{j}(x)
$$

therefore on account of the linear independence of $\phi_{1}(x), \cdots, \phi_{n}(x)$, $B_{q}=0(q=1,2, \cdots, n)$.

As an immediate deduction, we have

Corollary III. To linearly independent solutions of (1) correspond linearly independent solutions of (7); and conversely.

3. Turning now to the adjoint system $\left(1^{\prime}\right)$, we find similarly 
Theorem $\mathrm{I}^{\prime}$. Any solution $\left[v(y) ; v^{(1)}, \cdots, v^{(m)}\right]$ of $\left(1^{\prime}\right)$ is expressible in the form

$$
\begin{aligned}
v(y) & =\sum_{j=1}^{n} C_{j} \psi_{j}(y)+\sum_{i=1}^{m} D_{i} k\left(\xi_{i}, y\right), \\
v^{(p)} & =D_{p}
\end{aligned}
$$

where the $m+n$ constants $C_{1}, \cdots, C_{n} ; D_{1}, \cdots, D_{m}$ satisfy the system of $m+n$ linear algebraic equations:

$$
\begin{array}{cc}
\sum_{j=1}^{n} C_{j} \int_{a}^{b} \psi_{j}(s) K^{(p)}(s) d s-D_{p}+\sum_{i=1}^{m} D_{i}\left[K^{(p)}\left(\xi_{i}\right)\right. & \\
\left.+\int_{a}^{b} k\left(\xi_{i}, s\right) K^{(p)}(s) d s\right]=0 & (p=1,2, \cdots, m), \\
\sum_{i=1}^{m} D_{i} \phi_{q}\left(\xi_{i}\right)=0 & (q=1,2, \cdots, n) .
\end{array}
$$

Conversely, if $C_{1}, \cdots, C_{n} ; D_{1}, \cdots, D_{m}$ satisfy $\left(7^{\prime}\right)$, the system

$$
\left[v(y) ; v^{(1)}, \cdots, v^{(m)}\right]
$$

given by $\left(6^{\prime}\right)$ is a solution of $\left(1^{\prime}\right)$.

As before, suppose that we have a solution of $\left(1^{\prime}\right)$, and write $v^{(p)}=D_{p}$ as in $\left(6^{\prime}\right)$. Then

$$
v(y)=\sum_{i=1}^{n} D_{i} K\left(\xi_{i}, y\right)+\int_{a}^{b} v(s) K(s, y) d s .
$$

The condition $\left(4^{\prime}\right)$ that $\left(8^{\prime}\right)$ be possible gives the last set of $\left(7^{\prime}\right)$. Then writing out the value of $v(y)$ by $\left(5^{\prime}\right)$ and also applying the relation between $k(x, y)$ and $K(x, y)$,

$$
\begin{aligned}
v(y) & =\sum_{i=1}^{n} D_{i}\left[K\left(\xi_{i}, y\right)+\int_{a}^{b} K\left(\xi_{i}, s\right) k(s, y) d s\right]+\sum_{j=1}^{m} C_{j}^{\prime} \psi_{j}(y) \\
& =\sum_{i=1}^{n} D_{i} k\left(\xi_{i}, y\right)+\sum_{i=1}^{n} \sum_{j=1}^{m} D_{i} \psi_{j}\left(\xi_{i}\right) \psi_{j}(y)+\sum_{j=1}^{m} C_{j}^{\prime} \psi_{j}(y) \\
& =\sum_{i=1}^{n} D_{i} k\left(\xi_{i}, y\right)+\sum_{j=1}^{m} C_{j} \psi_{j}(y),
\end{aligned}
$$

as demanded by $\left(6^{\prime}\right)$. Substitution of the forms for $v(y)$ and $v^{(p)}$ in the second equation of $\left(1^{\prime}\right)$ now yields the first set of $\left(7^{\prime}\right)$. The converse is also readily shown, as in the previous case. We have further, in analogy with the earlier results:

CoRollary I. The correspondence between solutions of $\left(1^{\prime}\right)$ and solutions of $\left(7^{\prime}\right)$ is unique.

Corollary II. To a trivial solution of (1') corresponds a trivial solution of $\left(7^{\prime}\right)$; and conversely. 
Corollary III. To linearly independent solutions of $\left(1^{\prime}\right)$ correspond linearly independent solutions of $\left(7^{\prime}\right)$; and conversely.

4. We have thus reduced the study of solutions of (1), (1') to the study of solutions of the systems of linear homogeneous algebraic equations (7), $\left(7^{\prime}\right)$ respectively. The number of linearly independent solutions is in each case finite, being limited by the number of equations. Furthermore, the matrices of the coefficients of $(7)$ and $\left(7^{\prime}\right)$ are conjugate.* Thus the existence of a non-trivial solution of either implies the existence of a non-trivial solution of the other, and both possess the same number of linearly independent solutions. We restate these facts for $(1),\left(1^{\prime}\right)$ :

Theorem II. If either equation (1) or system (1') possesses a non-trivial solution, the other possesses a non-trivial solution; each possesses a finite number of linearly independent solutions, and the number is the same in both cases. $\dagger$

5 . If we apply the process used in the proof of Theorem I to the non-homogeneous equation (2), we obtain in place of (7) a non-homogeneous algebraic system for $A_{1}, \cdots, A_{m} ; B_{1}, \cdots, B_{n}$, the matrix of the coefficients being the same as in (7). If (1) possesses no non-trivial solutions, then (7) possesses no non-trivial solutions; hence the corresponding non-homogeneous algebraic system possesses one and only one solution, and therefore (2) possesses one and only one solution. Similarly, we see that if $\left(1^{\prime}\right)$ has no non-trivial solutions, $\left(2^{\prime}\right)$ has one and only one solution.

From the method just described by which the solutions may be obtained it is evident that in case the function $f(x)$ of (2), or the function $g(y)$ and constants $g^{(1)}, \cdots, g^{(m)}$ of $\left(2^{\prime}\right)$ contain parameters, the solutions will be continuous functions of all variables involved so long as the same is true of the given functions.

In equation (2), write $f(x)=K(x, y)$, regarding $y$ as a parameter; calling the solution $u(x)=Q(x, y)$, we see that in case (1) has no nontrivial solutions, there exists a continuous function $Q(x, y)$, such that

(9) $Q(x, y)=K(x, y)+\sum_{i=1}^{m} K^{(i)}(x) Q\left(\xi_{i}, y\right)+\int_{a}^{b} K(x, s) Q(s, y) d s$.

Again, writing $f(x)=K^{(p)}(x)$, we demonstrate the existence of continuous functions $Q^{(p)}(x)$, such that

$$
Q^{(p)}(x)=K^{(p)}(x)+\sum_{i=1}^{m} K^{(i)}(x) Q^{(p)}\left(\xi_{i}\right)+\int_{a}^{b} K(x, s) Q^{(p)}(s) d s .
$$

In the same manner, writing in $\left(2^{\prime}\right) g(y)=K(x, y), g^{(p)}=K^{(p)}(x)$,

* This fact and its consequences justify the designation of the system $\left(1^{\prime}\right)$ as adjoint to the equation (1).

† It may be readily shown that the number $\nu$ of linearly independent solutions satisfies the condition $n-m \leqq \nu \leqq n+m$. 
where $x$ is to be regarded as a parameter, we see that there exist continuous functions $R(x, y), R^{(p)}(x)$, such that

$\left(9^{\prime}\right) \quad R(x, y)=K(x, y)+\sum_{i=1}^{m} R^{(i)}(x) K\left(\xi_{i}, y\right)+\int_{a}^{b} R(x, s) K(s, y) d s$, $\left(10^{\prime}\right) \quad R^{(p)}(x)=K^{(p)}(x)+\sum_{i=1}^{m} R^{(i)}(x) K^{(p)}\left(\xi_{i}\right)+\int_{a}^{b} R(x, s) K^{(p)}(s) d s$.

The formulæ (9), (10), $\left(9^{\prime}\right),\left(10^{\prime}\right)$ are generalizations of the familiar resolvent formulæ of pure integral equations. It will shortly be seen that the functions $R$ and $Q$ are identical; postponing the proof of this fact, we show how the solutions of (2), $\left(2^{\prime}\right)$ may be expressed in terms of them. Starting with equation (2), which we know to possess a solution, compute directly by substitution of the right-hand side of (2) for $u(x)$ the expression

$$
\sum_{p=1}^{m} R^{(p)}(x) u\left(\xi_{p}\right)+\int_{a}^{b} R(x, s) u(s) d s ;
$$

after simplifying by use of $\left(9^{\prime}\right),\left(10^{\prime}\right)$, as in the case of pure integral equations, we find

$$
\begin{aligned}
\sum_{i=1}^{m} K^{(i)}(x) u\left(\xi_{i}\right)+\int_{a}^{b} K(x, s) & u(s) d s \\
& =\sum_{i=1}^{m} R^{(i)}(x) f\left(\xi_{i}\right)+\int_{a}^{b} R(x, s) f(s) d s .
\end{aligned}
$$

Hence the solution of (2) is

$$
u(x)=f(x)+\sum_{i=1}^{m} R^{(i)}(x) f\left(\xi_{i}\right)+\int_{a}^{b} R(x, s) f(s) d s .
$$

Similarly the solution of $\left(2^{\prime}\right)$ is given by the formulæ:

$$
\begin{aligned}
v(y) & =g(y)+\sum_{i=1}^{m} g^{(i)} Q\left(\xi_{i}, y\right)+\int_{a}^{b} g(s) Q(s, y) d s, \\
v^{(p)} & =g^{(p)}+\sum_{i=1}^{m} g^{(i)} Q^{(p)}\left(\xi_{i}\right)+\int_{a}^{b} g(s) Q^{(p)}(s) d s \\
& (p=1,2, \cdots, m) .
\end{aligned}
$$

We may prove the identity of the functions $Q, R$, as follows. Equations $\left(9^{\prime}\right),\left(10^{\prime}\right)$ together constitute a system of the form $\left(2^{\prime}\right)$, the unknowns being

$$
v(y)=R(x, y), \quad v^{(p)}=R^{(p)}(x),
$$

and the known functions

$$
g(y)=K(x, y), \quad g^{(p)}=K^{(p)}(x) .
$$

Trans. Am. Math. Soc. 9 
Writing out the solution in the form $\left(11^{\prime}\right)$,

$$
\begin{gathered}
R(x, y)=K(x, y)+\sum_{i=1}^{m} K^{(i)}(x) Q\left(\xi_{i}, y\right)+\int_{a}^{b} K(x, s) Q(s, y) d s, \\
R^{(p)}(x)=K^{(p)}(x)+\sum_{i=1}^{m} K^{(i)}(x) Q^{(p)}\left(\xi_{i}\right)+\int_{a}^{b} K(x, s) Q^{(p)}(s) d s \\
(p=1,2, \cdots, m) ;
\end{gathered}
$$

but the right-hand sides of these equations are equal respectively to the values given by (9) and (10) for $Q(x, y)$ and $Q^{(p)}(x)$.

6. We collect the results just deduced in the following theorem:

ThEOREM III. If (1), (1') have no non-trivial solutions, then there exists a resolvent system

to the kernel system

$$
\left[Q(x, y) ; Q^{(1)}(x), \cdots, Q^{(m)}(x)\right]
$$

$$
\left[K(x, y) ; K^{(1)}(x), \cdots, K^{(m)}(x)\right],
$$

satisfying the relations

$$
\begin{aligned}
& Q(x, y)=K(x, y)+\sum_{i=1}^{m} K^{(i)}(x) Q\left(\xi_{i}, y\right)+\int_{a}^{b} K(x, s) Q(s, y) d s, \\
& Q(x, y)=K(x, y)+\sum_{i=1}^{m} Q^{(i)}(x) K\left(\xi_{i}, y\right)+\int_{a}^{b b} Q(x, s) K(s, y) d s, \\
& Q^{(p)}(x)=K^{(p)}(x)+\sum_{i=1}^{m} K^{(i)}(x) Q^{(p)}\left(\xi_{i}\right)+\int_{a}^{b} K(x, s) Q^{(p)}(s) d s, \\
& Q^{(p)}(x)=K^{(p)}(x)+\sum_{i=1}^{m} Q^{(i)}(x) K^{(p)}\left(\xi_{i}\right)+\int_{a}^{o b} Q(x, s) K^{(p)}(s) d s \\
& (p=1,2, \cdots, m) .
\end{aligned}
$$

Furthermore, (2) possesses one and only one solution:

$$
u(x)=f(x)+\sum_{i=1}^{m} Q^{(i)}(x) f\left(\xi_{i}\right)+\int_{a}^{b} Q(x, s) f(s) d s ;
$$

and $\left(2^{\prime}\right)$ possesses one and only one solution:

$$
\begin{aligned}
v(y) & =g(y)+\sum_{i=1}^{m} g^{(i)} Q\left(\xi_{i}, y\right)+\int_{a}^{b} g(s) Q(s, y) d s, \\
v^{(p)} & =g^{(p)}+\sum_{i=1}^{m} g^{(i)} Q^{(p)}\left(\xi_{i}\right)+\int_{a}^{b} g(s) Q^{(p)}(s) d s \\
& \quad(p=1,2, \cdots, m) .
\end{aligned}
$$

7. We proceed now to study the case in which (1), (1') possess non-trivial solutions. Let a complete set of linearly independent solutions of (1) be

$$
u_{1}(x), \cdots, u_{\nu}(x) \text {. }
$$


By following out the form of proof used ordinarily in replacing a set of functions by a normal orthogonal set, we see that by proper linear combination the set of solutions may be made to satisfy the conditions:

$$
\int_{a}^{b} u_{\gamma}(s) u_{\rho}(s) d s+\sum_{i=1}^{m} u_{\gamma}\left(\xi_{i}\right) u_{\rho}\left(\xi_{i}\right)=\left\{\begin{array}{l}
0, \rho \neq \gamma . \\
1, \rho=\gamma .
\end{array}\right.
$$

Similarly, it is possible so to choose the $\nu$ linearly independent solutions of $\left(1^{\prime}\right)$ :

that

$$
\begin{aligned}
& {\left[\begin{array}{llll}
v_{1}(y) ; & v_{1}^{(1)}, & \cdots, & v_{1}^{(m)}
\end{array}\right]} \\
& {\left[v_{2}(y) ; \quad v_{2}^{(1)}, \cdots, \quad v_{2}^{(m)}\right] \text {, }} \\
& {\left[\begin{array}{llll}
v_{v}(y) ; & v_{\nu}^{(1)}, \cdots, & \left.v_{\nu}^{(m)}\right]
\end{array}\right.}
\end{aligned}
$$

$$
\int_{a}^{b} v_{\gamma}(s) v_{\rho}(s) d s+\sum_{i=1}^{m} v_{\gamma}^{(i)} v_{\rho}^{(i)}=\left\{\begin{array}{l}
0, \rho \neq \gamma . \\
1, \rho=\gamma .
\end{array}\right.
$$

Theorem IV.* Corresponding to any complete set of linearly independent solutions $u_{\gamma}(x)(\gamma=1,2, \cdots, \nu)$ of $(1)$, it is possible to select a set of collections of the form

$$
\left[U_{\gamma}(y) ; U_{\gamma}^{(1)}, \cdots, U_{\gamma}^{(m)}\right] \quad(\gamma=1,2, \cdots, \nu)
$$

each consisting of one continuous function and $m$ constants, such that

$$
\int_{a}^{b} u_{\gamma}(s) U_{\rho}(s) d s+\sum_{i=1}^{m} u_{\gamma}\left(\xi_{i}\right) U_{\rho}^{(i)}=\left\{\begin{array}{l}
0, \rho \neq \gamma . \\
1, \rho=\gamma .
\end{array}\right.
$$

Corresponding to any complete set of linearly independent solutions

$$
\left[v_{\gamma}(y) ; v_{\gamma}^{(1)}, \cdots, v_{\gamma}^{(m)}\right] \quad(\gamma=1,2, \cdots, \nu)
$$

of $\left(1^{\prime}\right)$, it is possible to select a set of continuous functions $V_{\gamma}(x)(\gamma=1,2$, $\cdots, \nu)$, such that

$$
\int_{a}^{b} V_{\gamma}(s) v_{\rho}(s) d s+\sum_{i=1}^{m} V_{\gamma}\left(\xi_{i}\right) v_{\rho}^{(i)}=\left\{\begin{array}{l}
0, \rho \neq \gamma . \\
1, \rho=\gamma .
\end{array}\right.
$$

It is sufficient to show that the theorem holds for a single complete set of solutions of each equation; for to any linear transformation of the functions $u_{\gamma}(x)$ we may evidently choose an appropriate linear transformation of the sets $\left[U_{\gamma}(y) ; U_{\gamma}^{(1)}, \cdots, U_{\gamma}^{(m)}\right]$, so that the relations (14) remain unaltered; and to any linear transformation of the sets $\left[v_{\gamma}(y) ; v_{\gamma}^{(1)}, \cdots, v_{\gamma}^{(m)}\right]$ we may choose a linear transformation of the functions $V_{\gamma}(x)$ so that the relations

* The conditions (13), $\left(13^{\prime}\right)$, corresponding to orthogonality, are not sufficient for the completion of the theory. We therefore establish in Theorem IV certain conditions analogous to "biorthogonality" of ordinary functions. 
$\left(14^{\prime}\right)$ remain unaltered. We therefore assume that the solutions have been so selected as to satisfy (13), $\left(13^{\prime}\right)$.

Slight consideration shows that the sets of $U$ 's may then be selected in a large variety of ways; for the proof of the theorem it will suffice to assign one choice satisfying the conditions. This is accomplished by taking

$$
U_{\rho}(y)=u_{\rho}(y), \quad U_{\rho}^{(p)}=u_{\rho}\left(\xi_{p}\right) .
$$

Similarly for the second part of the theorem take

$$
V_{\rho}(x)=\sum_{i=1}^{m} K^{(i)}(x) v_{\rho}^{(i)}+\int_{a}^{\triangleright b} K(x, s) v_{\rho}(s) d s .
$$

8. Theorem V. There exists a pseudo-resolvent system

$$
\left[Q(x, y) ; Q^{(1)}(x), \cdots, Q^{(m)}(x)\right]
$$

to the kernel system

such that

$$
\left[K(x, y) ; K^{(1)}(x), \cdots, K^{(m)}(x)\right]
$$

$$
\begin{aligned}
Q(x, y)=K(x, y)+ & \sum_{i=1}^{m} K^{(i)}(x) Q\left(\xi_{i}, y\right) \\
& +\int_{a}^{b} K(x, s) Q(s, y) d s-\sum_{\gamma=1}^{\nu} V_{\gamma}(x) v_{\gamma}(y), \\
Q(x, y)=K(x, y)+ & \sum_{i=1}^{m} Q^{(i)}(x) K\left(\xi_{i}, y\right) \\
& +\int_{a}^{b} Q(x, s) K(s, y) d s-\sum_{\gamma=1}^{\nu} u_{\gamma}(x) U_{\gamma}(y),
\end{aligned}
$$

$$
\begin{aligned}
& Q^{(p)}(x)=K^{(p)}(x)+\sum_{i=1}^{m} K^{(i)}(x) Q^{(p)}\left(\xi_{i}\right) \\
& \quad+\int_{a}^{b} K(x, s) Q^{(p)}(s) d s-\sum_{\gamma=1}^{\nu} V_{\gamma}(x) v_{\gamma}^{(p)}, \\
& \begin{aligned}
Q^{(p)}(x)=K^{(p)}(x)+\sum_{i=1}^{m} Q^{(i)}(x) K^{(p)}\left(\xi_{i}\right) \\
\quad+\int_{a}^{b} Q(x, s) K^{(p)}(s) d s-\sum_{\gamma=1}^{\nu} u_{\gamma}(x) U_{\gamma}^{(p)} \\
(p=1,2, \cdots, m) .
\end{aligned}
\end{aligned}
$$

The proof is similar to that used by the author in the case of pure integral equations.* Define the new kernel system:

* Loc. cit., p. 408. 


$$
\begin{aligned}
& L(x, y)=K(x, y)-\sum_{\gamma=1}^{\nu} V_{\gamma}(x) U_{\gamma}(y), \\
& L^{(p)}(x)=K^{(p)}(x)-\sum_{\gamma=1}^{\nu} V_{\gamma}(x) U_{\gamma}^{(p)} \quad(p=1,2, \cdots, m) .
\end{aligned}
$$

By (17), (1), (1'), (14), (14'), we have the identities:

$$
\begin{aligned}
& \int_{a}^{b} L(x, s) u_{\rho}(s) d s+\sum_{i=1}^{m} L^{(i)}(x) u_{\rho}\left(\xi_{i}\right)=u_{\rho}(x)-V_{\rho}(x) \\
& \quad(\rho=1,2, \cdots, \nu) ; \\
& \int_{a}^{b} v_{\rho}(s) L(s, y) d s+\sum_{i=1}^{m} v_{\rho}^{(i)} L\left(\xi_{i}, y\right)=v_{\rho}(y)-U_{\rho}(y), \\
& \int_{a}^{b} v_{\rho}(s) L^{(p)}(s) d s+\sum_{i=1}^{m} v_{\rho}^{(i)} L^{(p)}\left(\xi_{i}\right)=v_{\rho}^{(p)}-U_{\rho}^{(p)} \\
& (\rho=1,2, \cdots, \nu ; p=1,2, \cdots, m) .
\end{aligned}
$$

An equation of the same form as $\left(1^{\prime}\right)$ with the kernel system (17):

$$
u(x)=\sum_{i=1}^{m} L^{(i)}(x) u\left(\xi_{i}\right)+\int_{a}^{b} L(x, s) u(s) d s
$$

has no non-trivial solution. For from this equation follows by $\left(18^{\prime}\right)$, on applying to both sides the operation indicated by

the relation

$$
\int_{a}^{o} v_{\rho}(s) u(s) d s+\sum_{i=1}^{m} v_{\rho}^{(i)} u\left(\xi_{i}\right)
$$

$$
\int_{a}^{b} U_{\rho}(s) u(s) d s+\sum_{i=1}^{m} U_{\rho}^{(i)} u\left(\xi_{i}\right)=0 \quad(\rho=1,2, \cdots, \nu) .
$$

Substituting in the integral equation the values of the new kernel system and simplifying by (19), we find that

$$
u(x)=\sum_{i=1}^{m} K^{(i)}(x) u\left(\xi_{i}\right)+\int_{a}^{b} K(x, s) u(s) d s .
$$

Hence $u(x)$ is a linear combination of $u_{1}(x), \cdots, u_{v}(x)$ :

By (14),

$$
u(x)=c_{1} u_{1}(x)+\cdots+c_{\nu} u_{\nu}(x) .
$$

and by (19),

$$
\int_{a}^{b} U_{\rho}(s) u(s) d s+\sum_{i=1}^{m} U_{\rho}^{(i)} u\left(\xi_{i}\right)=c_{\rho} \quad(\rho=1,2, \cdots, \nu),
$$

$$
c_{\rho}=0 \quad(\rho=1,2, \cdots, \nu) .
$$

Thus $u(x)$ is identically zero, as we wished to show.

Since there are no non-trivial solutions for the kernel system 


$$
\left[L(x, y) ; L^{(1)}(x), \cdots, L^{(m)}(x)\right],
$$

there exists a resolvent system

such that

$$
\left[Q(x, y) ; Q^{(1)}(x), \cdots, Q^{(m)}(x)\right]
$$

$$
\begin{aligned}
& Q(x, y)=L(x, y)+\sum_{i=1}^{m} L^{(i)}(x) Q\left(\xi_{i}, y\right)+\int_{a}^{b} L(x, s) Q(s, y) d s, \\
& Q(x, y)=L(x, y)+\sum_{i=1}^{m} Q^{(i)}(x) L\left(\xi_{i}, y\right)+\int_{a}^{b} Q(x, s) L(s, y) d s, \\
& Q^{(p)}(x)=L^{(p)}(x)+\sum_{i=1}^{m} L^{(i)}(x) Q^{(p)}\left(\xi_{i}\right)+\int_{a}^{b} L(x, s) Q^{(p)}(s) d s, \\
& Q^{(p)}(x)=L^{(p)}(x)+\sum_{i=1}^{m} Q^{(i)}(x) L^{(p)}\left(\xi_{i}\right)+\int_{a}^{b} Q(x, s) L^{(p)}(s) d s \\
& \quad(p=1,2, \cdots, m) .
\end{aligned}
$$

To the first equation apply the operation indicated by

$$
\int_{a}^{b} v_{\rho}(s) Q(s, y) d s+\sum_{i=1}^{m} v_{\rho}^{(i)} Q\left(\xi_{i}, y\right) ;
$$

after simplifying by $\left(18^{\prime}\right)$, we find

$$
\begin{array}{r}
\int_{a}^{b} U_{\rho}(s) Q(s, y) d s+\sum_{i=1}^{m} U^{(i)} Q\left(\xi_{i}, y\right)=v_{\rho}(y)-U_{\rho}(y) \\
(\rho=1,2, \cdots, \nu) .
\end{array}
$$

Writing out the values of the $L$ 's in the first equation of (20), and simplifying by (21), we have the first equation of (16). An entirely analogous set of operations on the third equation of (20) yields the third equation of (16).

Again, operate on the second and fourth equations of (20) as indicated by

$$
\int_{a}^{b} Q(x, s) u_{\rho}(s) d s+\sum_{=1}^{m} Q^{(i)}(x) u_{\rho}\left(\xi_{i}\right)
$$

simplifying by (18), we have

$$
\begin{aligned}
\int_{a}^{b} Q(x, s) V_{\rho}(s) d s+\sum_{i=1}^{m} Q^{(i)}(x) V_{\rho}\left(\xi_{i}\right)=u_{\rho}(x)- & U_{\rho}(x) \\
& (\rho=1,2, \cdots, \nu) ;
\end{aligned}
$$

substituting this result in the forms obtained by writing out the values of the $L$ 's in the second and fourth equations of (20), we have the second and fourth equations of (16).

9. By means of the pseudo-resolvent system we can complete the theory of the solution in the non-homogeneous case, as follows: 
THEOREM VI. In order that (2) possess a solution it is necessary and sufficient that

$$
\int_{a}^{b} v_{\gamma}(s) f(s) d s+\sum_{i=1}^{m} v_{\gamma}^{(i)} f\left(\xi_{i}\right)=0 \quad(\gamma=1,2, \cdots, \nu) .
$$

If this condition is satisfied, every solution may be written in the form:

$$
u(x)=f(x)+\sum_{i=1}^{m} Q^{(i)}(x) f\left(\xi_{i}\right)+\int_{a}^{b} Q(x, s) f(s) d s+\sum_{\gamma=1}^{\nu} c_{\gamma} u_{\gamma}(x) .
$$

TheOREM VI'. In order that (2') possess a solution it is necessary and sufficient that

$$
\int_{a}^{b} g(s) u_{\gamma}(s) d s+\sum_{i=1}^{m} g^{(i)} u_{\gamma}\left(\xi_{i}\right)=0 \quad(\gamma=1,2, \cdots, \nu) .
$$

If this condition is satisfied, every solution may be written in the form:

$$
\begin{array}{r}
v(y)=g(y)+\sum_{i=1}^{m} g^{(i)} Q\left(\xi_{i}, y\right)+\int_{a}^{b} g(s) Q(s, y) d s+\sum_{\gamma=1}^{\nu} c_{\gamma} v_{\gamma}(y), \\
v^{(p)}=g^{(p)}+\sum_{i=1}^{m} g^{(i)} Q^{(p)}\left(\xi_{i}\right)+\int_{a}^{b} g(s) Q^{(p)}(s) d s+\sum_{\gamma=1}^{\nu} c_{\gamma} v_{\gamma}^{(p)} \\
\quad(p=1,2, \cdots, m) .
\end{array}
$$

The proof of these two theorems follows the same lines as that of the corresponding developments in the case of pure equations.*

* W. A. Hurwitz, loc. cit., p. 409.

Cornell University, July, 1914. 